\title{
MONOTONE ITERATIVE TECHNIQUE FOR SEMILINEAR ELLIPTIC SYSTEMS
}

\author{
A. S. VATSALA AND JIE YANG
}

Received 27 September 2004 and in revised form 23 January 2005

We develop monotone iterative technique for a system of semilinear elliptic boundary value problems when the forcing function is the sum of Caratheodory functions which are nondecreasing and nonincreasing, respectively. The splitting of the forcing function leads to four different types of coupled weak upper and lower solutions. In this paper, relative to two of these coupled upper and lower solutions, we develop monotone iterative technique. We prove that the monotone sequences converge to coupled weak minimal and maximal solutions of the nonlinear elliptic systems. One can develop results for the other two types on the same lines. We further prove that the linear iterates of the monotone iterative technique converge monotonically to the unique solution of the nonlinear BVP under suitable conditions.

\section{Introduction}

Semilinear systems of elliptic equations arise in a variety of physical contexts, specially in the study of steady-state solutions of time-dependent problems. See $[1,4,5]$, for example. Existence and uniqueness of classical solutions of such systems by monotone method has been established in $[2,4]$. Using generalized monotone method, the existence and uniqueness of coupled weak minimal and maximal solutions for the scalar semilinear elliptic equation has been established in [3]. They have utilized the existence and uniqueness result of weak solution of the linear equation from [1]. In [3], the authors have considered coupled upper and lower solutions and have obtained natural sequences as well as alternate sequences which converge to coupled weak minimal and maximal solutions of the scalar semilinear elliptic equation.

In this paper, we develop generalized monotone method combined with the method of upper and lower solutions for the system of semilinear elliptic equations. For this purpose, we have developed a comparison result for the system of semilinear elliptic equations which yield the result of the scalar comparison theorem of [3] as a special case. One can derive analog results for the other two types of coupled weak upper and lower solutions on the same lines. We develop two main results related to two different types of coupled weak upper and lower solutions of the nonlinear semilinear elliptic systems. 
We obtain natural as well as intertwined monotone sequences which converge uniformly to coupled weak minimal and maximal solutions of the semilinear elliptic system. Further using the comparison theorem for the system, we establish the uniqueness of the weak solutions for the nonlinear semilinear elliptic systems. The existence of the solution of the linear system has been obtained as a byproduct of our main results.

\section{Preliminaries}

In this section, we present some known comparison results, existence and uniqueness results related to scalar semilinear elliptic BVP without proofs. See [1, 3] for details.

Consider the semilinear elliptic BVP

$$
\begin{gathered}
\mathscr{L} u=F(x, u) \quad \text { in } U, \\
u=0 \quad \text { on } \partial U(\text { in the sense of trace }),
\end{gathered}
$$

where $U$ is an open, bounded subset of $R^{m}$ and $u: \bar{U} \rightarrow \mathbb{R}$ is unknown, $u=u(x)$. Here $F: U \rightarrow \mathbb{R}$ is known. $F \in L^{2}(U), F(x, u)$ is a Caratheodory function, that is, $F(\cdot, u)$ is measurable for all $u \in \mathbb{R}$ and $F(x, \cdot)$ is continuous a.e. $x \in U . \mathscr{L}$ denotes a second-order partial differential operator with the divergence form

$$
\mathscr{L} u=-\sum_{i, j=1}^{m}\left(a_{i j}(x) u_{x_{i}}\right)_{x_{j}}+c(x) u
$$

for given coefficient functions $a_{i j}(x), c(x) \in L^{\infty}(U)(i=1,2, \ldots, m)$. We assume the symmetry condition $a_{i j}=a_{j i}(i, j=1, \ldots, m), c(x) \geq 0$, and the partial differential operator $\mathscr{L}$ is uniformly elliptic such that there exists a constant $\theta>0$ such that

$$
\sum_{i, j=1}^{m} a_{i j}(x) \xi_{i} \xi_{j} \geq \theta|\xi|^{2}
$$

for a.e. $x \in U$ and all $\xi \in \mathbb{R}^{m}$.

We recall the following definitions for future use.

Definition 2.1. (i) The bilinear form $B[\cdot, \cdot]$ associated with the divergence form of the elliptic operator $\mathscr{L}$ defined by (2.2) is

$$
B[u, v]=\int_{U}\left[\sum_{i, j=1}^{m} a_{i j}(x) u_{x_{i}} v_{x_{j}}+c(x) u v\right] d x
$$

for $u, v \in H_{0}^{1}(U)$, where $H_{0}^{1}(U)$ is a Sobolev space $W_{0}^{1,2}(U)$. 
(ii) We say that $u \in H_{0}^{1}(U)$ is a weak solution of the boundary value problem (2.1) if

$$
B[u, v]=(F, v)
$$

for all $v \in H_{0}^{1}(U)$, where $(\cdot, \cdot)$ denotes the inner product in $L^{2}(U)$.

Definition 2.2. The function $\alpha_{0} \in H^{1}(U)$ is said to be a weak lower solution of (2.1) if, $\alpha_{0} \leq 0$ on $\partial U$ and

$$
\int_{U}\left[\sum_{i, j=1}^{m} a_{i j}(x) \alpha_{0, x_{i}} v_{x_{j}}+c(x) \alpha_{0} v\right] d x \leq \int_{U} F\left(x, \alpha_{0}\right) v d x
$$

for each $v \in H_{0}^{1}(U), v \geq 0$. If the inequalities are reversed, then $\alpha_{0}$ is said to be a weak upper solution of (2.1).

In order to discuss the results on monotone iterative technique, we need to consider the existence and uniqueness of weak solutions of linear boundary value problems. The result on the existence of weak solutions for the linear BVP can be obtained from the Lax-Milgram theorem which is stated below. In the following theorem, we assume that $H$ is a real Hilbert space, with norm $\|\cdot\|$ and inner product $(\cdot, \cdot)$, we let $\langle\cdot, \cdot\rangle$ denote the pairing of $H$ with its dual space.

Theorem 2.3 (the Lax-Milgram theorem). Assume that $B: H \times H \rightarrow \mathbb{R}$ is a bilinear mapping, for which there exist constants $\alpha, \beta>0$ such that

(i) $|B[u, v]| \leq \alpha\|u\|\|v\|, u, v \in H$;

(ii) $\beta\|u\|^{2} \leq B[u, u], u \in H$.

Also assume that $F: H \rightarrow \mathbb{R}$ is a bounded linear functional on $H$.

Then there exists a unique element $u \in H$ such that

$$
B[u, v]=\langle f, v\rangle
$$

for all $v \in H$.

The following theorem proves the unique solution of the linear BVP, which is $[3$, Theorem 5.2.4].

Theorem 2.4. Consider the linear BVP

$$
\begin{gathered}
\mathscr{L} u=h(x) \quad \text { in } U, \\
u=0 \quad \text { on } \partial U(\text { in the sense of trace }) .
\end{gathered}
$$

Then there exists a unique solution $u \in H_{0}^{1}(U)$ for the linear $B V P(2.8)$ provided $0<c^{*} \leq$ $c(x)$ a.e. in $U$ and $h \in L^{2}(U)$.

The next theorem is a comparison theorem, a modified version of which is needed in our main results. This is [3, Theorem 5.2.5]. 
Theorem 2.5. Let $\alpha_{0}, \beta_{0}$ be weak lower and upper solutions of (2.1). Suppose further that $F$ satisfies

$$
F\left(x, u_{1}\right)-F\left(x, u_{2}\right) \leq K\left(u_{1}-u_{2}\right)
$$

whenever $u_{1} \geq u_{2}$ a.e. for $x \in U$ and $K(x)>0$ for $x \in U$. Then, if $0<c-K \in L^{1}(U)$,

$$
\alpha_{0}(x) \leq \beta_{0}(x) \text { in } U \text { a.e. }
$$

The following corollary is the special case of Theorem 2.5.

Corollary 2.6. For $p \in H^{1}(U)$ satisfying

$$
\int_{U}\left[\sum_{i, j=1}^{m} a_{i j}(x) p_{x_{i}} v_{x_{j}}+c(x) p v\right] d x \leq 0
$$

for each $v \in H_{0}^{1}(U), v \geq 0$ a.e. and $p \leq 0$ on $\partial U, p(x) \leq 0$ in $U$ a.e. provided $c(x)>0$.

The next two theorems [1] are needed to prove that a bounded sequence in a Hilbert space contains a weakly, uniformly convergent subsequence.

Theorem 2.7 (weak compactness). Let $X$ be a reflexive Banach space and suppose that the sequence $\left\{u_{k}\right\}_{k=1}^{\infty} \in X$ is bounded. Then there exist a subsequence $\left\{u_{k_{j}}\right\}_{j=1}^{\infty} \subseteq\left\{u_{k}\right\}_{k=1}^{\infty}$ and $u \in X$ such that $\left\{u_{k_{j}}\right\}_{j=1}^{\infty}$ converges weakly to $u \in X$.

Theorem 2.8 (the Ascoli-Arzela theorem). Suppose that $\left\{f_{k}\right\}_{k=1}^{\infty}$ is a sequence of realvalued functions defined on $\mathbb{R}^{n}$ such that

$$
\left|f_{k}(x)\right| \leq M \quad\left(k=1,2, \ldots, x \in \mathbb{R}^{n}\right)
$$

for some constant $M$, and the $\left\{f_{k}\right\}_{k=1}^{\infty}$ are uniformly equicontinuous, then there exist a subsequence $\left\{f_{k_{j}}\right\}_{j=1}^{\infty} \subseteq\left\{f_{k}\right\}_{k=1}^{\infty}$ and a continuous function $f$ such that $f_{k_{j}} \rightarrow f$ uniformly on compact subset of $\mathbb{R}^{n}$.

\section{Main results}

In this section, we develop monotone iterative technique for system of semilinear elliptic BVP. The results of [3] will be a special case of our results for the scalar semilinear elliptic BVP. form

We first consider the following system of semilinear elliptic BVP in the divergence

$$
\begin{gathered}
\mathscr{L} u=f(x, u)+g(x, u) \quad \text { in } U, \\
u=0 \text { on } \partial U(\text { in the sense of trace }),
\end{gathered}
$$

where $u: \bar{U} \rightarrow \mathbb{R}^{N}, \mathscr{L} u=\left(\mathscr{L}^{1} u^{1}, \mathscr{L}^{2} u^{2}, \ldots, \mathscr{L}^{N} u^{N}\right)$, and $\mathscr{L}^{k} u^{k}=-\left(\sum_{i, j=1}^{m} a_{i j}^{k}(x) u_{x_{i}}^{k}\right)_{x_{j}}+$ $c^{k}(x) u^{k}$ with the bilinear form $B\left[u^{k}, v^{k}\right]=\int_{U}\left(\sum_{i, j=1}^{m} a_{i j}^{k}(x) u_{x_{i}}^{k} v_{x_{j}}^{k}+c^{k}(x) u^{k} v^{k}\right) d x$ for $k=$ $1,2, \ldots, N$. Here $f, g: U \times \mathbb{R}^{N} \rightarrow \mathbb{R}^{N}$ are Caratheodory functions. Other assumptions on $a_{i j}^{k}, c^{k}$ are the same as for $a_{i j}, c$ in Section 2 . 
In this paper, here and throughout, we assume all the inequalities to be componentwise unless otherwise stated.

In order to develop monotone iterative technique for the BVP (3.1), we need to prove the following comparison Lemma 3.1 relative to the elliptic system

$$
\begin{gathered}
\mathscr{L} u=F(x, u) \quad \text { in } U, \\
u=0 \quad \text { on } \partial U(\text { in the sense of trace }),
\end{gathered}
$$

where assumption for $\mathscr{L} u, \mathscr{L}^{k} u^{k}, B\left[u^{k}, v^{k}\right]$ are the same as they are in (3.1).

LeMmA 3.1. Let $\alpha_{0}, \beta_{0}$ be weak lower and upper solutions of (3.2) when $F: U \times \mathbb{R}^{N} \rightarrow$ $\mathbb{R}^{N}, u \in H_{0}^{1}(U)$. Suppose further that $F(x, u)$ is quasimonotone nondecreasing in $u$ for each component $k$ and satisfies

$$
F^{k}\left(x, u^{1}, u^{2}, \ldots, u^{N}\right)-F^{k}\left(x, v^{1}, v^{2}, \ldots, v^{N}\right) \leq K^{k} \sum_{i=1}^{N}\left(u^{i}-v^{i}\right)
$$

whenever $u \geq v$ a.e. for $x \in U$ and $K^{k}>0$ for $k=1,2, \ldots, N$. Then, if $0<c^{k}-N \bar{K} \in L^{1}(U)$, where $\bar{K}=\max K^{k}$ for $k=1,2, \ldots, N$,

$$
\alpha_{0}^{k}(x) \leq \beta_{0}^{k}(x) \quad \text { in } U \text { a.e. for } k=1,2, \ldots, N \text {. }
$$

Proof. From the definition of weak lower and upper solutions, we get

$$
\int_{U}\left[\sum_{i, j=1}^{m} a_{i j}^{k}(x)\left(\alpha_{0, x_{i}}^{k}-\beta_{0, x_{i}}^{k}\right) v_{x_{j}}^{k}+c^{k}(x)\left(\alpha_{0}^{k}-\beta_{0}^{k}\right) v^{k}\right] d x \leq \int_{U}\left[F^{k}\left(x, \alpha_{0}\right)-F^{k}\left(x, \beta_{0}\right)\right] v^{k} d x
$$

for each $v^{k} \in H_{0}^{1}(U), v^{k} \geq 0$ a.e. and $k=1,2, \ldots, N$. Choose $v^{k}=\left(\alpha_{0}^{k}-\beta_{0}^{k}\right)^{+} \in H_{0}^{1}(U)$, $v^{k} \geq 0$ a.e.

Since

$$
\left(\alpha_{0}^{k}-\beta_{0}^{k}\right)_{x_{j}}^{+}= \begin{cases}\alpha_{0, x_{j}}^{k}-\beta_{0, x_{j}}^{k} & \text { a.e. on } \alpha_{0}^{k}>\beta_{0}^{k} \\ 0 & \text { a.e. on } \alpha_{0}^{k} \leq \beta_{0}^{k}\end{cases}
$$

using the ellipticity condition (2.3), and (3.3), we integrate (3.5) on the region where $\alpha_{0}^{k}>\beta_{0}^{k}$, for $k=1,2, \ldots, N$, and we have

$$
\int_{\alpha_{0}>\beta_{0}}\left(\theta^{k}\left|\alpha_{0, x_{i}}^{k}-\beta_{0, x_{i}}^{k}\right|^{2}+c^{k}(x)\left|\alpha_{0}^{k}-\beta_{0}^{k}\right|^{2}\right) d x \leq \int_{\alpha_{0}>\beta_{0}} K^{k} \sum_{i=1}^{N}\left(\alpha_{0}^{i}-\beta_{0}^{i}\right)\left(\alpha_{0}^{k}-\beta_{0}^{k}\right) d x .
$$


We have $N$ such inequalities for $k=1,2, \ldots, N$. When we add all $N$ inequalities together, we obtain

$$
\begin{aligned}
& \int_{\alpha_{0}>\beta_{0}}\left(\sum_{k=1}^{N} \theta^{k}\left|\alpha_{0, x_{i}}^{k}-\beta_{0, x_{i}}^{k}\right|^{2}+\sum_{k=1}^{N} c^{k}(x)\left|\alpha_{0}^{k}-\beta_{0}^{k}\right|^{2}\right) d x \\
& \leq \int_{\alpha_{0}>\beta_{0}} \sum_{k=1}^{N}\left|\alpha_{0}^{k}-\beta_{0}^{k}\right|\left[\sum_{k=1}^{N} K^{k}\left(\alpha_{0}^{k}-\beta_{0}^{k}\right)\right] d x, \\
& \int_{\alpha_{0}>\beta_{0}}\left(\sum_{k=1}^{N} \theta^{k}\left|\alpha_{0, x_{i}}^{k}-\beta_{0, x_{i}}^{k}\right|^{2}+\sum_{k=1}^{N} c^{k}(x)\left|\alpha_{0}^{k}-\beta_{0}^{k}\right|^{2}\right) d x \leq \int_{\alpha_{0}>\beta_{0}} N \bar{K} \sum_{k=1}^{N}\left|\alpha_{0}^{k}-\beta_{0}^{k}\right|^{2} d x, \\
& \int_{\alpha_{0}>\beta_{0}} \sum_{k=1}^{N}\left(\theta^{k}\left|\alpha_{0, x_{i}}^{k}-\beta_{0, x_{i}}^{k}\right|^{2}+\left(c^{k}(x)-N \bar{K}\right)\left|\alpha_{0}^{k}-\beta_{0}^{k}\right|^{2}\right) d x \leq 0 .
\end{aligned}
$$

From our assumption, the integrand is nonnegative. Hence, the only possibility to keep our inequalities hold true is that the domain of integration is an empty set. Hence, we have $\alpha_{0} \leq \beta_{0}$ a.e. in $U$.

If, in (3.2), $F(x, u)=A(x) u$, where $A(x)$ is an $N \times N$ matrix, we have the following corollary for the linear system.

Corollary 3.2. Let $F(x, u)=A(x) u$ in (3.2) and all the assumptions of Lemma 3.1 hold, further let

$$
A(x) u-A(x) v \leq\left(K^{1}, K^{2}, \ldots, K^{N}\right)^{\prime}\left(\sum_{i=1}^{N}\left(u^{i}-v^{i}\right)\right)
$$

whenever $u \geq v$ a.e. for $x \in U$ and $K^{k}>0$ for $k=1,2, \ldots, N$. Then, if $0<c^{k}-N K^{k} \in$ $L^{1}(U)$, where $K^{k}=\max \left(\left|a_{k 1}\right|,\left|a_{k 2}\right|, \ldots,\left|a_{k N}\right|\right)$ for $k=1,2, \ldots, N$,

$$
\alpha_{0}^{k}(x) \leq \beta_{0}^{k}(x) \quad \text { in } U \text {, a.e. for } k=1,2, \ldots, N \text {. }
$$

The next corollary is a special application of Lemma 3.1.

Corollary 3.3. For $p^{k} \in H^{1}(U), k=1,2, \ldots, N$, satisfying

$$
\int_{U} \sum_{k=1}^{N}\left[\sum_{i, j=1}^{m} a_{i j}^{k}(x) p_{x_{i}}^{k} v_{x_{j}}^{k}+c_{0}^{k}(x) p^{k} v^{k}\right] d x \leq 0
$$

for each $v^{k} \in H_{0}^{1}(U), v^{k} \geq 0$ a.e. and $p^{k} \leq 0$ on $\partial U$, then $p^{k}(x) \leq 0$ in $U$ a.e. provided that $c_{0}^{k}>0$ for $x \in U, k=1,2, \ldots, N$.

Next, we define two types of coupled weak lower and upper solutions of (3.1). In order to avoid monotony, our main results are developed relative to these two types of coupled weak lower and upper solutions only. 
Definition 3.4. Relative to the BVP (3.1), the functions $\alpha_{0}, \beta_{0} \in H^{1}(U)$ are said to be

(i) coupled weak lower and upper solutions of type I if

$$
\begin{aligned}
& B\left[\alpha_{0}^{k}, v^{k}\right] \leq\left(f^{k}\left(x, \alpha_{0}\right)+g^{k}\left(x, \beta_{0}\right), v^{k}\right), \\
& B\left[\beta_{0}^{k}, v^{k}\right] \geq\left(f^{k}\left(x, \beta_{0}\right)+g^{k}\left(x, \alpha_{0}\right), v^{k}\right),
\end{aligned}
$$

for each $v^{k} \in H_{0}^{1}(U), v^{k} \geq 0$ a.e. in $U$ and $k=1,2, \ldots, N$;

(ii) coupled weak lower and upper solutions of type II if

$$
\begin{aligned}
& B\left[\alpha_{0}^{k}, v^{k}\right] \leq\left(f^{k}\left(x, \beta_{0}\right)+g^{k}\left(x, \alpha_{0}\right), v^{k}\right), \\
& B\left[\beta_{0}^{k}, v^{k}\right] \geq\left(f^{k}\left(x, \alpha_{0}\right)+g^{k}\left(x, \beta_{0}\right), v^{k}\right),
\end{aligned}
$$

for each $v^{k} \in H_{0}^{1}(U), v^{k} \geq 0$ a.e. in $U$ and $k=1,2, \ldots, N$.

We are now in a position to prove the first main result on monotone method for the system of elliptic BVP (3.1).

Theorem 3.5. Assume that

(A1) $\alpha_{0}, \beta_{0} \in H^{1}(U)$ are the coupled weak lower and upper solutions of type I with $\alpha_{0}(x) \leq$ $\beta_{0}(x)$ a.e. in $U \times \mathbb{R}^{N}$;

(A2) $f, g: U \times \mathbb{R}^{N} \rightarrow \mathbb{R}^{N}$ are Caratheodory functions such that $f^{k}(x, u)$ is nondecreasing in each component $u^{i}, g^{k}(x, u)$ is nonincreasing in each component $u^{i}$ for $x \in U$ a.e. where $i, k=1,2, \ldots, N$;

(A3) $c^{k}(x) \geq c_{k}^{*}>0$ in $U$ a.e. and for any $\eta, \mu \in H^{1}\left(U \times \mathbb{R}^{N}\right)$ with $\alpha_{0} \leq \eta, \mu \leq \beta_{0}$, the function $h^{k}(x)=f^{k}(x, \eta)+g^{k}(x, \mu) \in L^{2}(U)$ for $k=1,2, \ldots, N$.

Then for any solution $u(x)$ of $B V P(3.1)$ with $\alpha_{0}(x) \leq u(x) \leq \beta_{0}(x)$, there exist monotone sequences $\left\{\alpha_{n}(x)\right\},\left\{\beta_{n}(x)\right\} \in H_{0}^{1}\left(U \times \mathbb{R}^{N}\right)$ such that $\alpha_{n}^{k} \rightarrow \rho^{k}, \beta_{n}^{k} \rightarrow \gamma^{k}$ weakly in $H_{0}^{1}(U)$ as $n \rightarrow \infty$ and $(\rho, \gamma)$ are coupled weak minimal and maximal solutions of (3.1), respectively, that is,

$$
\begin{array}{ll}
\mathscr{L}^{k} \rho^{k}=f^{k}(x, \rho)+g^{k}(x, \gamma) & \text { in } U, \rho^{k}=0 \text { on } \partial U, \\
\mathscr{L}^{k} \gamma^{k}=f^{k}(x, \gamma)+g^{k}(x, \rho) & \text { in } U, \gamma^{k}=0 \text { on } \partial U
\end{array}
$$

for $k=1,2, \ldots, N$.

Note. Here and in Theorem 3.8, when we say that $\rho, \gamma$ are coupled weak solutions means that they satisfy the following variational form:

$$
\begin{aligned}
& B\left[\rho^{k}, v^{k}\right]=\int_{U}\left[f^{k}(x, \rho)+g^{k}(x, \gamma)\right] v^{k} d x, \\
& B\left[\gamma^{k}, v^{k}\right]=\int_{U}\left[f^{k}(x, \gamma)+g^{k}(x, \rho)\right] v^{k} d x .
\end{aligned}
$$

Proof. Consider the linear BVP

$$
\begin{array}{ll}
\mathscr{L}^{k} \alpha_{n+1}^{k}=f^{k}\left(x, \alpha_{n}\right)+g^{k}\left(x, \beta_{n}\right) & \text { in } U, \alpha_{n+1}^{k}=0 \text { on } \partial U, \\
\mathscr{L}^{k} \beta_{n+1}^{k}=f^{k}\left(x, \beta_{n}\right)+g^{k}\left(x, \alpha_{n}\right) & \text { in } U, \beta_{n+1}^{k}=0 \text { on } \partial U,
\end{array}
$$


where $n=0,1, \ldots$. The variational forms associated with (3.16) are

$$
\begin{aligned}
& B\left[\alpha_{n+1}^{k}, v^{k}\right]=\int_{U}\left[f^{k}\left(x, \alpha_{n}\right)+g^{k}\left(x, \beta_{n}\right)\right] v^{k} d x, \\
& B\left[\beta_{n+1}^{k}, v^{k}\right]=\int_{U}\left[f^{k}\left(x, \beta_{n}\right)+g^{k}\left(x, \alpha_{n}\right)\right] v^{k} d x,
\end{aligned}
$$

for all $v^{k} \in H_{0}^{1}(U), v^{k} \geq 0$ a.e. in $U$ for $k=1,2, \ldots, N$.

We want to show that the weak solutions $\alpha_{n}, \beta_{n}$ of (3.16) are uniquely defined and satisfy

$$
\alpha_{0} \leq \alpha_{1} \leq \cdots \leq \alpha_{n} \leq \beta_{n} \leq \cdots \leq \beta_{1} \leq \beta_{0} \text { a.e. in } U .
$$

For each $n \geq 1$, if we have $\alpha_{0} \leq \alpha_{n} \leq \beta_{n} \leq \beta_{0}$, then by hypothesis $(A 3), h_{1}^{k}(x)=f^{k}\left(x, \alpha_{n}\right)+$ $g^{k}\left(x, \beta_{n}\right) \in L^{2}(U), h_{2}^{k}(x)=f^{k}\left(x, \beta_{n}\right)+g^{k}\left(x, \alpha_{n}\right) \in L^{2}(U)$, and $c^{k}(x) \geq c_{k}^{*}>0$. Hence, Theorem 2.4 implies that BVP (3.16) has unique weak solution $\alpha_{n}^{k}$ and $\beta_{n}^{k}$ for $k=1$, $2, \ldots, N$.

In order to show that (3.18) is true, we first prove that $\alpha_{1}^{k} \geq \alpha_{0}^{k}$ a.e. in $U$ for each $k$ th component. Now let $p^{k}=\alpha_{0}^{k}-\alpha_{1}^{k}$ so that $p^{k} \leq 0$ on $\partial U$ and for $v^{k} \in H_{0}^{1}(U), v^{k} \geq 0$ a.e. in $U$, by the definition of type I of coupled weak lower and upper solutions, we have

$$
\begin{aligned}
B\left[p^{k}, v^{k}\right] & =B\left[\alpha_{0}^{k}, v^{k}\right]-B\left[\alpha_{1}^{k}, v^{k}\right] \\
& \leq \int_{U}\left[f^{k}\left(x, \alpha_{0}\right)+g^{k}\left(x, \beta_{0}\right)\right] v^{k} d x-\int_{U}\left[f^{k}\left(x, \alpha_{0}\right)+g^{k}\left(x, \beta_{0}\right)\right] v^{k} d x=0 .
\end{aligned}
$$

Hence, by Corollary 2.6, $p^{k} \leq 0$ in $U$ a.e., that is, $\alpha_{0}^{k} \leq \alpha_{1}^{k}$ in $U$ a.e. Similarly, we can show that $\beta_{1}^{k} \leq \beta_{0}^{k}$ a.e. in $U$, where $k=1,2, \ldots, N$.

Assume, for some fixed $n>1, \alpha_{n} \leq \alpha_{n+1}$ and $\beta_{n} \geq \beta_{n+1}$ a.e. in $U$. Now consider $p^{k}=$ $\alpha_{n+1}^{k}-\alpha_{n+2}^{k}$, with $p^{k}=0$ on $\partial U$, and using the monotone properties of $f, g$, we get

$$
B\left[p^{k}, v^{k}\right]=\int_{U}\left[f^{k}\left(x, \alpha_{n}\right)+g^{k}\left(x, \beta_{n}\right)-f^{k}\left(x, \alpha_{n+1}\right)-g^{k}\left(x, \beta_{n+1}\right)\right] v^{k} d x \leq 0 .
$$

By Corollary 2.6, we get $\alpha_{n+1}^{k} \leq \alpha_{n+2}^{k}$ a.e. in $U$. Similarly, we can show that $\beta_{n+1}^{k} \geq \beta_{n+2}^{k}$ a.e. in $U$ componentwise. Hence, using the induction argument, we get $\alpha_{n-1}^{k} \leq \alpha_{n}^{k}, \beta_{n-1}^{k} \geq \beta_{n}^{k}$ a.e. in $U$ for all $n \geq 1$.

Now we want to show that $\alpha_{1} \leq \beta_{1}$ a.e. in $U$. Consider $p^{k}=\alpha_{1}^{k}-\beta_{1}^{k}$ and $p^{k}=0$ on $\partial U$. Since $\alpha_{0} \leq \beta_{0}$, by the monotone properties of $f, g$, we have

$$
B\left[p^{k}, v^{k}\right]=\int_{U}\left[f^{k}\left(x, \alpha_{0}\right)+g^{k}\left(x, \beta_{0}\right)-f^{k}\left(x, \beta_{0}\right)-g^{k}\left(x, \alpha_{0}\right)\right] v^{k} d x \leq 0 .
$$

Hence, $\alpha_{1}^{k} \leq \beta_{1}^{k}$ a.e. in $U$ for $k=1,2, \ldots, N$ by Corollary 2.6.

Assume $\alpha_{n}^{k} \leq \beta_{n}^{k}$ a.e. in $U$ for some fixed $n>1$. We can also prove $\alpha_{n+1}^{k} \leq \beta_{n+1}^{k}$ a.e. in $U$ using similar argument. By induction, (3.18) holds for $n \geq 1$.

Since monotone sequences $\left\{\alpha_{n}\right\},\left\{\beta_{n}\right\} \in H_{0}^{1}\left(U \times \mathbb{R}^{N}\right)$, there exist pointwise limits for each component $k$, where $k=1,2, \ldots, N$. That is,

$$
\lim _{n \rightarrow \infty} \alpha_{n}^{k}(x)=\rho^{k}(x) \quad \text { a.e. in } U, \quad \lim _{n \rightarrow \infty} \beta_{n}^{k}(x)=\gamma^{k}(x) \quad \text { a.e. in } U,
$$


where $\rho^{k}, \gamma^{k} \in H_{0}^{1}(U)$, since a Hilbert space is a Banach space which is a complete, normed linear space.

For each $n \geq 1$, we note that for each $v^{k} \in H_{0}^{1}(U), \alpha_{n}^{k}$ satisfies

$$
\int_{U}\left[\sum_{i, j=1}^{m} a_{i j}^{k}(x)\left(\alpha_{n}^{k}\right)_{x_{i}} v_{x_{j}}+c^{k}(x) \alpha_{n}^{k} v^{k}\right] d x=\int_{U}\left[f^{k}\left(x, \alpha_{n-1}\right)+g^{k}\left(x, \beta_{n-1}\right)\right] v^{k} d x .
$$

We now use the ellipticity condition and the fact that $c^{k}(x) \geq c_{k}^{*}(x)>0$ with $v^{k}=\alpha_{n}^{k}$ to get

$$
\int_{U}\left[\theta^{k}\left(\alpha_{n, x}^{k}\right)^{2}+c_{k}^{*}(x)\left(\alpha_{n}^{k}\right)^{2}\right] d x \leq \int_{U}\left[f^{k}\left(x, \alpha_{n-1}\right)+g^{k}\left(x, \beta_{n-1}\right)\right] v^{k} d x .
$$

Since the integrand on the right-hand side belongs to $L^{2}(U)$, we obtain the estimate

$$
\sup _{n}\left\|\alpha_{n}^{k}\right\|_{H_{0}^{1}(U)}<\infty
$$

Hence, there exists a subsequence $\left\{\alpha_{n_{i}}^{k}\right\}$ which converges weakly to $\rho^{k}(x)$ in $H_{0}^{1}(U)$ by Theorem 2.7. Similarly, we can show that $\sup _{n}\left\|\beta_{n}^{k}\right\|_{H_{0}^{1}(U)}<\infty$. Hence, there exists a subsequence $\left\{\beta_{n_{i}}^{k}\right\}$ which converges weakly to $\gamma^{k}(x)$ in $H_{0}^{1}(U)$ using Theorem 2.7.

Sequence $\left\{\alpha_{n}^{k}(x)\right\}$ maps $U$ into $\mathbb{R}$ for each $k=1,2, \ldots, N$. It is easy through contradiction method to show that for each $\varepsilon>0$, there exists $\delta>0$ such that $|x-y|<\delta$ implies that $\left\|\alpha_{n}^{k}(x)-\alpha_{n}^{k}\left(x_{0}\right)\right\|_{W^{1,2}(U)}<\varepsilon$ for $x, y \in U$. Hence, $\left\{\alpha_{n}^{k}(x)\right\}$ is equicontinuous on $U$. Similarly, we can show that $\left\{\beta_{n}^{k}(x)\right\}$ is also equicontinuous on $U$. Then by the AscoliArzela theorem, the subsequences $\left\{\alpha_{n_{i}}^{k}\right\},\left\{\beta_{n_{i}}^{k}\right\}$ converge uniformly on $U$. Since both of the sequences $\left\{\alpha_{n}^{k}(x)\right\},\left\{\beta_{n}^{k}(x)\right\}$ are monotone, the entire sequences converge uniformly and weakly to $\rho^{k}(x), \gamma^{k}(x)$, respectively, on $U$ for $k=1,2, \ldots, N$. Therefore, taking the limit as $n \rightarrow \infty$ for (3.17), we obtain

$$
\begin{aligned}
& B\left[\rho^{k}, v^{k}\right]=\int_{U}\left[f^{k}(x, \rho)+g^{k}(x, \gamma)\right] v^{k} d x, \\
& B\left[\gamma^{k}, v^{k}\right]=\int_{U}\left[f^{k}(x, \gamma)+g^{k}(x, \rho)\right] v^{k} d x .
\end{aligned}
$$

Hence, $\rho, \gamma$ are the coupled weak solutions of (3.1). Finally, we want to prove that $\rho$ and $\gamma$ are the coupled weak minimal and maximal solutions of (3.1). That is, if $u$ is any weak solution of (3.1) such that $\alpha_{0}(x) \leq u(x) \leq \beta_{0}(x)$ a.e. in $U \times \mathbb{R}^{N}$, then the following claim will be true. For $k=1,2, \ldots, N$,

$$
\alpha_{0}^{k}(x) \leq \rho^{k}(x) \leq u^{k}(x) \leq \gamma^{k}(x) \leq \beta_{0}^{k}(x) \text { a.e. in } U \text {. }
$$

To prove that for any fixed $n \geq 1, \alpha_{n}^{k}(x) \leq u^{k}(x) \leq \beta_{n}^{k}(x)$ a.e. in $U$, we assume that for some fixed $n \geq 1, \alpha_{n}^{k}(x) \leq u^{k}(x) \leq \beta_{n}^{k}(x)$ a.e. in $U$ is true, since $\alpha_{0}(x) \leq u(x) \leq \beta_{0}(x)$ is claimed from the hypothesis. Let $p^{k}=\alpha_{n+1}^{k}-u^{k}$, with $p^{k}=0$ on $\partial U$. Using the monotone properties of $f, g$, we obtain

$$
B\left[p^{k}, v^{k}\right]=\int_{U}\left[f^{k}\left(x, \alpha_{n}\right)+g^{k}\left(x, \beta_{n}\right)-f^{k}(x, u)-g^{k}(x, u)\right] v^{k} d x \leq 0 .
$$


Hence, by Corollary 2.6, $\alpha_{n+1}^{k} \leq u^{k}$ a.e. in $U$. In a similar way, we obtain $u^{k} \leq \beta_{n+1}^{k}$. By induction, $\alpha_{n}^{k}(x) \leq u^{k}(x) \leq \beta_{n}^{k}(x)$ a.e. in $U$ for all $n \geq 1$. Now taking the limit of $\alpha_{n}^{k}, \beta_{n}^{k}$ as $n \rightarrow \infty$, we get (3.27). This completes the proof.

Remark 3.6. (i) When $N=1$, the results of Theorem 3.5 yield the scalar result of [3], which is [3, Theorem 5.2.1].

(ii) In (3.1), if $g(x, u) \equiv 0, f(x, u)$ is not nondecreasing in some $u^{k}$ components, where $k=1,2, \ldots, N$, then we can construct $\bar{f}^{k}(x, u)=f^{k}(x, u)+d^{k} u^{k}$ which is nondecreasing in each $u^{k}$ with $d^{k} \geq 0$. Let $\bar{g}^{k}(x, u)=-d^{k} u^{k}$ which is nonincreasing in $u^{k}$. Then we can solve the BVP

$$
\mathscr{L}^{k} u^{k}=-\left(\sum_{i, j=1}^{m} a_{i j}^{k}(x) u_{x_{i}}^{k}\right)_{x_{j}}+c^{k}(x) u^{k}=\bar{f}^{k}(x, u)+\bar{g}^{k}(x, u),
$$

where $(\bar{f})^{k}(x, u)$ is nondecreasing in each $u^{l}, \bar{g}^{k}(x, u)$ is nonincreasing in each $u^{l}$ for $l, k=$ $1,2, \ldots, N$. Assume that the type- $I$ coupled weak upper lower solutions of (3.1) are also the type-I coupled weak upper lower solutions of the new constructed elliptic BVP (3.29), then Theorem 3.5 still can be applied to (3.29) and the solutions of (3.29) will be the solutions for (3.1).

(iii) In (3.1), if $f(x, u) \equiv 0, g(x, u)$ is not nonincreasing in some $u^{k}$ components, where $k=1,2, \ldots, N$, then we can construct $\bar{g}^{k}(x, u)=g^{k}(x, u)-d^{k} u^{k}$ which is nondecreasing in each $u^{k}$ with $d^{k} \geq 0$. Let $\bar{f}^{k}(x, u)=d^{k} u^{k}$ which is nondecreasing in $u^{k}$. Then we can solve the BVP

$$
\mathscr{L}^{k} u^{k}=-\left(\sum_{i, j=1}^{m} a_{i j}^{k}(x) u_{x_{i}}^{k}\right)_{x_{j}}+c^{k}(x) u^{k}=\bar{f}^{k}(x, u)+\bar{g}^{k}(x, u),
$$

where $\bar{f}^{k}(x, u)$ is nondecreasing in each $u^{l}, \bar{g}^{k}(x, u)$ is nonincreasing in each $u^{l}$ for $l, k=$ $1,2, \ldots, N$. Assume that the type $I$ coupled with upper lower solutions of (3.1) are also the type- $I$ coupled weak upper lower solutions of the new constructed elliptic BVP (3.30), then apply Theorem 3.5 to (3.30) and get the solutions we need for (3.1).

(iv) Other varieties on the properties of $f(x, u), g(x, u)$ such as $f(x, u)$ is not nondeceasing in every $u^{k}$ component and $g(x, u)$ is not nonincreasing in every $u^{k}$ component, we can always use the idea in (ii), (iii) to solve the new constructed elliptic BVP under suitable assumption of coupled upper and lower solutions for the newly constructed problem.

The following corollary is to show the uniqueness of the solution for (3.1).

Corollary 3.7. Assume, in addition to the conditions of Theorem 3.5, $f$ and $g$ satisfy

$$
\begin{gathered}
f^{k}\left(x, u^{1}, u^{2}, \ldots, u^{N}\right)-f^{k}\left(x, v^{1}, v^{2}, \ldots, v^{N}\right) \leq N_{1} \sum_{i=1}^{N}\left(u^{i}-v^{i}\right), \\
g^{k}\left(x, u^{1}, u^{2}, \ldots, u^{N}\right)-g^{k}\left(x, v^{1}, v^{2}, \ldots, v^{N}\right) \geq-N_{2} \sum_{i=1}^{N}\left(u^{i}-v^{i}\right),
\end{gathered}
$$


where $u \geq v, N_{1}, N_{2}>0, C-N\left(N_{1}+N_{2}\right)>0$ a.e. in $U$ where $C=\min c^{k}(x), x \in U$ and $k=1,2, \ldots, N$.

Then $\rho^{k}=u^{k}=\gamma^{k}$ is the unique weak solution of (3.1).

Proof. Since we have $\rho \leq \gamma$, let $p^{k}=\gamma^{k}-\rho^{k}$ and $p^{k}=0$ on $\partial U$, we get

$$
\begin{aligned}
B\left[p^{k}, v\right] & =\int_{U}\left(\sum_{i, j=1}^{m} a_{i j}^{k} p_{x_{i}}^{k} v_{x_{j}}+c^{k} p^{k} v\right) d x \\
& =\int_{U}\left[f^{k}(x, \gamma)+g^{k}(x, \rho)-f^{k}(x, \rho)-g^{k}(x, \gamma)\right] v d x \\
& \leq \int_{U}\left(N_{1}+N_{2}\right)\left(\sum_{i=1}^{N}\left(\gamma^{i}-\rho^{i}\right)\right) v d x .
\end{aligned}
$$

We have $N$ such inequalities for $k=1,2, \ldots, N$. Adding $N$ of them together, we obtain

$$
\begin{aligned}
& \int_{U}\left(\sum_{k=1}^{N} \sum_{i, j=1}^{m}\left(a_{i j}^{k} p_{x_{i}}^{k} v_{x_{j}}\right)+\sum_{k=1}^{N}\left(c^{k} p^{k} v\right)\right) d x \leq \int_{U} N\left(N_{1}+N_{2}\right)\left(\sum_{k=1}^{N} p^{k}\right) v d x, \\
& \int_{U} \sum_{k=1}^{N} \sum_{i, j=1}^{m}\left(a_{i j}^{k} p_{x_{i}}^{k} v_{x_{j}}\right)+\sum_{k=1}^{N}\left[c^{k}-N\left(N_{1}+N_{2}\right)\right] p^{k} v d x \leq 0, \\
& \int_{U} \sum_{k=1}^{N}\left[\sum_{i, j=1}^{m}\left(a_{i j}^{k} p_{x_{i}}^{k} v_{x_{j}}\right)+\left[c^{k}-N\left(N_{1}+N_{2}\right)\right] p^{k} v\right] d x \leq 0 .
\end{aligned}
$$

However,

$$
\begin{aligned}
\int_{U} \sum_{k=1}^{N} & {\left[\sum_{i, j=1}^{m}\left(a_{i j}^{k} p_{x_{i}}^{k} v_{x_{j}}\right)+\left[C-N\left(N_{1}+N_{2}\right)\right] p^{k} v\right] d x } \\
& \leq \int_{U} \sum_{k=1}^{N}\left[\sum_{i, j=1}^{m}\left(a_{i j}^{k} p_{x_{i}}^{k} v_{x_{j}}\right)+\left[c^{k}-N\left(N_{1}+N_{2}\right)\right] p^{k} v\right] d x \leq 0 .
\end{aligned}
$$

By assumption, $C-N\left(N_{1}+N_{2}\right)>0$, we have $c^{k}-N\left(N_{1}+N_{2}\right)>0$ for $k=1,2, \ldots, N$. Using Corollary 3.3, we have $\gamma^{k} \leq \rho^{k}$ for $k=1,2, \ldots, N$. Hence, (3.1) has unique weak solution.

We also have similar results for coupled weak lower upper solutions of type II. We state the result below with a brief sketch of the proof.

Theorem 3.8. Assume that

(A1) $\alpha_{0}, \beta_{0} \in H^{1}(U)$ are coupled weak lower and upper solutions of type II with $\alpha_{0} \leq \beta_{0}$ a.e. in $U \times \mathbb{R}^{N}$;

(A2) $f, g: U \times \mathbb{R}^{N} \rightarrow \mathbb{R}^{N}$ are Caratheodory functions such that $f^{k}(x, u)$ is nondecreasing in each component $u^{i}, g^{k}(x, u)$ is nonincreasing in $u^{i}$ for $x \in U$ a.e. where $i, k=$ $1,2, \ldots, N$;

(A3) $c^{k}(x) \geq c_{k}^{*}>0$ in $U$ a.e. and for any $\eta, \mu \in H^{1}(U)$ with $\alpha_{0} \leq \eta, \mu \leq \beta_{0}$, the function $h^{k}(x)=f^{k}(x, \eta)+g^{k}(x, \mu) \in L^{2}(U)$. 
Then for any solution $u(x)$ of BVP (3.1) provided $\alpha_{0}(x) \leq u(x) \leq \beta_{0}(x), \alpha_{0} \leq \beta_{1}, \alpha_{1} \leq \beta_{0}$, there exist intertwining alternating sequences $\left\{\alpha_{2 n}(x), \beta_{2 n+1}(x)\right\}$ and $\left\{\beta_{2 n}(x), \alpha_{2 n+1}(x)\right\} \in$ $H_{0}^{1}\left(U \times \mathbb{R}^{N}\right)$ satisfying

$$
\alpha_{0} \leq \beta_{1} \leq \cdots \leq \alpha_{2 n} \leq \beta_{2 n+1} \leq u \leq \alpha_{2 n+1} \leq \beta_{2 n} \leq \cdots \leq \alpha_{1} \leq \beta_{0}
$$

such that $\left\{\alpha_{2 n}^{k}(x), \beta_{2 n+1}^{k}(x)\right\} \rightarrow \rho^{k}$ and $\left\{\beta_{2 n}^{k}(x), \alpha_{2 n+1}^{k}(x)\right\} \rightarrow \gamma^{k}$ weakly in $H_{0}^{1}(U)$ as $n \rightarrow \infty$ and $(\rho, \gamma)$ are coupled weak minimal and maximal solutions of (3.1), respectively,

$$
\begin{aligned}
& \mathscr{L} \rho^{k}=f^{k}(x, \gamma)+g^{k}(x, \rho) \quad \text { in } U, \rho^{k}=0 \text { on } \partial U, \\
& \mathscr{L} \gamma^{k}=f^{k}(x, \rho)+g^{k}(x, \gamma) \quad \text { in } U, \gamma^{k}=0 \text { on } \partial U,
\end{aligned}
$$

for $k=1,2, \ldots, N$.

Proof. The sequences $\left\{\alpha_{n}\right\},\left\{\beta_{n}\right\}$ are defined as the coupled weak solutions in the following system of linear elliptic BVP:

$$
\begin{aligned}
& \mathscr{L}^{k} \alpha_{n+1}^{k}=f^{k}\left(x, \beta_{n}\right)+g^{k}\left(x, \alpha_{n}\right) \text { in } U, \alpha_{n+1}^{k}=0 \text { on } \partial U, \\
& \mathscr{L}^{k} \beta_{n+1}^{k}=f^{k}\left(x, \alpha_{n}\right)+g^{k}\left(x, \beta_{n}\right) \text { in } U, \beta_{n+1}^{k}=0 \text { on } \partial U .
\end{aligned}
$$

Since we have $\alpha_{0}(x) \leq u(x) \leq \beta_{0}(x)$ and $\alpha_{0} \leq \beta_{1}, \alpha_{1} \leq \beta_{0}$, let $p^{k}=\beta_{1}^{k}-\alpha_{1}^{k}$ and $p^{k}=0$ on $\partial U$. We get $B\left[p^{k}, v^{k}\right]=\int_{U}\left[f^{k}\left(x, \alpha_{0}\right)+g^{k}\left(x, \beta_{0}\right)-f^{k}\left(x, \beta_{0}\right)-g^{k}\left(x, \alpha_{0}\right)\right] v^{k} d x \leq 0$, using the monotone nature of $f$ and $g$. By Corollary 2.6, we have $\beta_{1} \leq \alpha_{1}$. Similarly, we can prove $\beta_{1} \leq u \leq \alpha_{1}$. Hence, we obtain

$$
\alpha_{0} \leq \beta_{1} \leq u \leq \alpha_{1} \leq \beta_{0}
$$

Our aim is to prove

$$
\alpha_{0} \leq \beta_{1} \leq \alpha_{2} \leq \beta_{3} \leq \cdots \leq \alpha_{2 n} \leq \beta_{2 n+1} \leq u \leq \alpha_{2 n+1} \leq \beta_{2 n} \leq \cdots \leq \alpha_{3} \leq \beta_{2} \leq \alpha_{1} \leq \beta_{0} .
$$

For that purpose, we assume that for some fixed $n \geq 1,(3.40)$ is true. We want to show that (3.40) also holds for $n+1$. Let $p^{k}=\beta_{2 n+1}^{k}-\alpha_{2 n+2}^{k}$, then

$$
B\left[p^{k}, v^{k}\right]=\int_{U}\left[f^{k}\left(x, \alpha_{2 n}\right)+g^{k}\left(x, \beta_{2 n}\right)-f^{k}\left(x, \beta_{2 n+1}\right)-g^{k}\left(x, \alpha_{2 n+1}\right)\right] v^{k} d x \leq 0
$$

because $\alpha_{2 n} \leq \beta_{2 n+1}, \beta_{2 n} \geq \alpha_{2 n+1}$ and the monotone properties of $f, g$. Hence, $\beta_{2 n+1}^{k} \leq$ $\alpha_{2 n+2}^{k}$ for all $k=1,2, \ldots, N$. 
Similarly, we can prove that $\alpha_{2 n+2} \leq \beta_{2 n+3}, \beta_{2 n+3} \leq u, \beta_{2 n+1} \leq u, \beta_{2 n+2} \leq \alpha_{2 n+1}, \alpha_{2 n+2} \leq$ $\beta_{2 n+2}$, and $u \leq \alpha_{2 n+2}$ by a similar reasoning. Hence, (3.40) is true for $n+1$ also.

Notice that $\alpha_{2 n}^{k}, \alpha_{2 n+1}^{k}, \beta_{2 n}^{k}, \beta_{2 n+1}^{k} \in H_{0}^{1}(U)$ and hence, arguing as in the proof of Theorem 3.5 with appropriate modification, we obtain that

$$
\begin{array}{rc}
\alpha_{2 n}^{k} \rightarrow \rho^{k} & \text { uniformly and weakly in } H_{0}^{1}(U), \\
\beta_{2 n+1}^{k} \rightarrow \rho^{k} & \text { uniformly and weakly in } H_{0}^{1}(U), \\
\alpha_{2 n+1}^{k} \rightarrow \gamma^{k} & \text { uniformly and weakly in } H_{0}^{1}(U), \\
\beta_{2 n}^{k} \rightarrow \gamma^{k} & \text { uniformly and weakly in } H_{0}^{1}(U) .
\end{array}
$$

For the variational form of (3.37), when $n=2 k$, as $n \rightarrow \infty$, we get

$$
B\left[\gamma^{k}, v^{k}\right]=\int_{U}\left[f^{k}(x, \gamma)+g^{k}(x, \rho)\right] v^{k} d x
$$

When $n=2 k+1$, as $n \rightarrow \infty$, we get

$$
B\left[\rho^{k}, v^{k}\right]=\int_{U}\left[f^{k}(x, \rho)+g^{k}(x, \gamma)\right] v^{k} d x
$$

For the variational form of (3.38), when $n=2 k$, as $n \rightarrow \infty$, we get

$$
B\left[\rho^{k}, v^{k}\right]=\int_{U}\left[f^{k}(x, \rho)+g^{k}(x, \gamma)\right] v^{k} d x
$$

When $n=2 k+1$, as $n \rightarrow \infty$, we get

$$
B\left[\gamma^{k}, v^{k}\right]=\int_{U}\left[f^{k}(x, \gamma)+g^{k}(x, \rho)\right] v^{k} d x
$$

Hence, when $n \rightarrow \infty$ in (3.37) and (3.38), we obtain

$$
\begin{aligned}
& B\left[\gamma^{k}, v^{k}\right]=\int_{U}\left[f^{k}(x, \gamma)+g^{k}(x, \rho)\right] v^{k} d x, \\
& B\left[\rho^{k}, v^{k}\right]=\int_{U}\left[f^{k}(x, \rho)+g^{k}(x, \gamma)\right] v^{k} d x .
\end{aligned}
$$

This proves that $\rho^{k} \leq u^{k} \leq \gamma^{k}$ a.e. in $U$, where $\rho$ and $\gamma$ are coupled weak minimal and maximal solutions of (3.1).

Note. We can write a similar remark for Theorem 3.8 on the same lines as Remark 3.6. We avoid this remark due to monotony.

For the uniqueness of solution for (3.1) with type II coupled weak lower upper solutions, we have following corollary. 
Corollary 3.9. Assume, in addition to the conditions of Theorem 3.8, $f^{k}$ and $g^{k}$ satisfy one-sided Lipschitz condition of the form

$$
\begin{gathered}
f^{k}\left(x, u^{1}, u^{2}, \ldots, u^{N}\right)-f^{k}\left(x, v^{1}, v^{2}, \ldots, v^{N}\right) \geq-N_{1} \sum_{i=1}^{N}\left(u^{i}-v^{i}\right), \\
g^{k}\left(x, u^{1}, u^{2}, \ldots, u^{N}\right)-g^{k}\left(x, v^{1}, v^{2}, \ldots, v^{N}\right) \leq N_{2} \sum_{i=1}^{N}\left(u^{i}-v^{i}\right),
\end{gathered}
$$

where $u \geq v, N_{1}, N_{2}>0, C-N\left(N_{1}+N_{2}\right)>0$ a.e. in $U$, where $C=\min c^{k}(x), x \in U$ and $k=1,2, \ldots, N$.

Then $\rho^{k}=u^{k}=\gamma^{k}$ is the unique weak solution of (3.1).

The proof of Corollary 3.9 follows on the same lines as the proof of Corollary 3.7.

\section{References}

[1] L. C. Evans, Partial Differential Equations, Graduate Studies in Mathematics, vol. 19, American Mathematical Society, Rhode Island, 1998.

[2] G. S. Ladde, V. Lakshmikantham, and A. S. Vatsala, Monotone Iterative Techniques for Nonlinear Differential Equations, Monographs, Advanced Texts and Surveys in Pure and Applied Mathematics, vol. 27, Pitman (Advanced Publishing Program), Massachusetts, 1985.

[3] V. Lakshmikantham and S. Köksal, Monotone Flows and Rapid Convergence for Nonlinear Partial Differential Equations, Series in Mathematical Analysis and Applications, vol. 7, Taylor \& Francis, London, 2003.

[4] C. V. Pao, Nonlinear Parabolic and Elliptic Equations, Plenum Press, New York, 1992.

[5] H. L. Smith, Spatial ecology via reaction-diffusion equations, Bull. Amer. Math. Soc. (N.S.) 41 (2004), no. 4, 551-557.

A. S. Vatsala: Department of Mathematics, University of Louisiana at Lafayette, Lafayette, LA 70504-1010, USA

E-mail address: vatsala@louisiana.edu

Jie Yang: Department of Mathematics, University of Louisiana at Lafayette, Lafayette, LA 705041010, USA

E-mail address: jxy5278@louisiana.edu 